\title{
XXXIII.
}

\section{Aus den Verhandlungen der XII. Tagung der Deutschen Pathologischen Gesellschaft, gehalten zu Kiel vom 23.-25. April 1908.}

\author{
Referent: Dr. Max Koch (Berlin).
}

Von den dreitägigen Verhandlungen der Deutschen Pathologischen Gesellschaft waren in diesem Jabre fast zwei volle Tage allgemeinen und speziellen Erörterungen über die Geschwülste gewidmet, worüber im folgenden berichtet werden soll. Bei dem

\section{Referat: „Ueber die Genese des Carcinoms":}

kamen zunächst und zum ersten Male in der Deutschen Pathologischen Gesellschaft die Herren Apolant und Ehrlich-Frankfurt a. M. als Vertreter der experimentellen Krebsforschung zum Wort, wobei ersterer die auf experimentellem Wege gewonnenen Resultate, soweit sie sich für die Genese des Carcinoms verwerten lassen, in histologischer Hinsicht zur Sprache brachte, wäbrend letzterer sich die biologische Seite des Problems vorbehalten hatte.

Herr Apolant führte ungefähr folgendes aus: Die experimentelle Tieronkologie hat uns neue Probleme crschlossen, alte in nevem Iichte erscheinen lassen. Unsere Versuchstiere zeigen eine verschiedene Disposition zu Tumoren hinsichtlich der Spezies, der Organe und der Art der Geschwülste. So finden sich bei Kaninchen und Meersohweinchen nur selten Tumoren. Beispielsweise konnten unter all' den zahlreichen im Frankfurter Serumprüfungsinstitut verwendeten Meerschweinchen nur zweimal Geschwülste, und zwar cystische Adenome der Mamma gefunden werden. Verhältnismässig häufig sind dagegen Geschwülste bej Mäusen und Ratten und zwar bei ersteren mehr solche epithelialer, bei letzteren bindegewebiger Natur, wie ja die Disposition bestimmter Tiergattungen für bestimmte Geschwülste durch die Beobachtung von Marianne Plehn an den Schilddrüsenkrebsen der Salmoniden bewiesen wird. Wenn sich anch Rückschlüsse in ätiologischer Hinsicht aus dem Befallensein bestimmter Tierarten noch nicht ergeben haben, so hat uns das Studium dieser Geschwülste doch die Beherrschung: der Uebertragung gelehrt. Indem das Tier gewissermassen als Nährboden fungiert, 
ist man auch von der Beobachtung individueller Einzelgeschwülste zu einer Stammesgeschichte einzelner Geschwulstarten oder des Carcinoms fortgeschritten. Dass es sich bei den Mäusetumoren wirklich um von der Brustdrüse ansgehende Carcinome und nicht, wie verschiedene Autoren meinen, um Endotheliome handelt, dafür sprechen nach Apolant folgende Gründe: 1. das Freisein der männlichen Mäuse von diesen Tumoren, 2. das Vorkommen der Tumoren nur an Stellen, wo eine Beziehung zur Brustdrüse gegeben, 3. der Nachweis direkten Zusammenhanges mit der Brustdrüse, 4. die exakte Widerlegung aller gegenteiligen Argumente. Der einfachste Typus der Mäusetumoren ist das Adenom, das entweder azinös oder papillös sein kann und häufig hämorrhagische Degeneration aufweist. Die Umwandlung in Carcinom findet multizentrisch statt, wie durch Serienschnitte bewiesen. Erstaunlich ist dabei die Intensität des Prozesses. Da nur gewisse Teile in Carcinom umgewandelt werden, so finden sich histologisch die grössten Gegensätze, neben enormen Krebsmassen Gebiete ron zierlichstem adenomatösen Bau. Das Verhalten des Bindegewebes an den Stellen der Unwandlung in Krebs spricht gegen die Ribbertsche Theorie, indem entzündliche Infiltrationen meist fehlen, ja die Stellen der Carcinomentwicklung sich durch besondere Stromaarmut auszeichnen. Ueberhaupt ist Stromaarmut ein Charakteristikum dieser Geschwülste, häufig ist die Adventitia der Gefässe das einzige Stroma, woraus die Aehnlichkeit mit Endotheliomen resultiert. Durch zahllose Mitosen weisen ganze Gebiete eine hellere Färbung auf. In den Imp fserien wird zunächst der Typus der ursprünglichen Geschwulst (hämorrhagischer Tumor, alveolärer, papillärer, steinbildende: Krebs, Carcinoma solidum, reticulatum usw.) beibehalten, aber nur bis zur Aenderung der Virulenz, d. h. der Wachstumsschnelligkeit, später findet sich immer Carcinoma solidum reticulatum. Die verschiedenen Typen sind Ausdruck der Virulenz oder das histologische Bild ist eine Fanktion der Virulenz. Die biologischen Eigenschaften der Tumorzellen und die Resistenz des Tieres bedingen die Besonderheiten. Aenderungen in diesen beiden führen zu histologischen Strukturveränderungen. Das aus Ehrlichs Veröffentlichungen bekannte Chondrom der Maus, welches ursprünglich hämorthagisch war, verlor seine angiotaktischen Eigenschaiten, wenn das Impfmaterial auf Eis aufbewahrt wurde, und zeigte auch bei subkutaner Impfung frühzeitige Nekrosen, wie sonst bei intraperitonealer lmpfung. Dieselben Veränderungen traten auf bei vorheriger Immunisierung. Unter ähnlichen Bedingungen wurde für das Carcinom ein Rückschlag in den azinösen Bau des Adenoms konstatiert.

Die vor drei Jahren von Ehrlich und Apolant zuerst beobachtete Umwandlung von Carcinom in Sarkom wurde im Frankfurter Institut 3 mal beobachtet und von vier verschiedenen Seiten von auswärts bestätigt. Bei den drei Frankfurter Beobachtungen handelte es sich je einmal um eine Umwandlung in Spindel-, Rundzellen- und polymorphzelliges Sarkom. Die Umwandlung wurde 2 mal konstatiert in einer Primärgeschwulst, schon in der ersten oder erst in der 67. Generation. Auch im zeitlichen Ablauf der Umwandlung fanden sich grosse Differenzen, so fand in einem Falle die Umwandlung über 20 Generationen von Mischtumoren statt. Die sarkomatöse Komponente stammt nicht aus dem mitverimpften Stroma, dessen Zugrundegehen durch Jensen und Basb ford einwandsfrei erwiesen. Es muss daher mit Ehrlich ein formbestimmender Einfluss des 
Epithels auf das Bindegewebe (wie in den Metastasen) angenommen werden. Eine Bestätigung für die Ehrlichsche Theorie bietet die Lewinsche Beobachtung, dass sich über einem sekundären Sarkom ein Kankroid entwickelte. Dieser Vorgang ist kein Beweis für eine parasitäre Aetiologie, wohl aber für eine spezifiscbe chemische Wirkung des Epithels. Bei der Sarkomentwicklung handelt es sich vielleicht um einen gesetzmässigen Vorgang und vielleicht stellt das Sarkom überhaupt das natürliche Ende jeden Carcinoms dar, was beim Menschen infolge des vorzeitigen Todes der Träger nur äusserst selten zur Beobachtung kommt. Für die Carcinomzellen ist demnach eine ähnliche Unsterblichkeit anzunehmen wie für die Protozoen.

Zum Schluss berichtet A. noch über eine nicht abgeschlossene Beobachtung. Bei einem Carcinomstamme mit kolossaler Virulenzsteigerung, der über 85 Generationen in $4^{1} / 2$ Jahren weitergezüchtet wurde und immer als medulläres Carcinom gewachsen war, trat in der Peripherie der Krebsnester eine Umwandlung der Epithelzellen in Spindelzellen auf. Zugleich fand sich hier ein eigentümliches rarefiziertés Aussehen und eine Auflösung in Zellen. So ketzerisch es wäre, eine direkte Umwandlung ron Carcinom in Sarkom anzunehmen, so muss doch hervorgehoben werden, dass hier eine Trennung der Carcinom- und Sarkomkomponente nicht mehr möglich ist und der Anschein einer vollständigen Auflösung in Sarkomzelien erweckt wird. Auf ähnliche Prozesse bei menschlichen Krebsen müsste jedenfalls geachtet werden. Mit der Sarkomentwicklung wird bei Mäusen die Struktur stationär, bei Rattensarkomen wurde in der 2. Geveration Umwandlung aus Spindelzellensarkom in Lymphosarkom und umgekehrt beobachtet, was von Lewin bestätigt wurde. Als allgemeines Resultat der experimentellen Krebsforschung in bistologischer Beziehung ergibt sich, dass alle histologischen Strukturbilder abhängen

1. von den biologischen Eigenschaften der Tumorzellen und

2. von der Resistenz des Tieres.

Herr Ehrlich (Frankfurt a. M.) bespricht sodann die biologische Seite der Ergebnisse der experimentellen Krebsforschung. Von dieser hofft er, das sie, wenn sie auch von entgegengesetzter Seite den Spaten angesetzt babe, doch dereinst mit der pathologischen Anatomie sich in der Mitte des Tunnels die Hand reichen werde.

Die Verimpfung von Spontantumoren auf gesunde Mäuse ist nur wenig erfolgreich, besonders bei den sogenannten hämorrhagischen Tumoren. In Bashfords Institut hat Gierke mit der Stückenimpfung (vermittels der Impfnadel) bei letzteren günstigere Resultate erzielt, bei den übrigen Tumoren ist die Breiimpfung vorzuziehen. Die resultatlos geimpften Mäuse (sogenannte Nuller nach Ehrlich) erwiesen sich als immun. Die Angangsziffer schwankt zwischen $1 / 4^{-1 / 2}$ pCt. bei Spontantumoren, bis zu $100 \mathrm{pCt}$. bei Tumoren mit gesteigerter Virulenz. Bei gleichzeitiger Impfung ein und derselben Maus mit Tumoren von verschiedener Virulenz gestaltet, sich das Resultat genau dementsprechend, gleichviel ob zwei oder vier Tumoren verschiedener Angangsziffer verimpft wurden. Die Resistenz jeder einzelnen Maus ist also den verschiedenen Tumoren gegenüber verschieden. Man kann daher die Geschwulstresistenz messen 
und stellt jede Maus eine Skala ron verschiedener Resistenz dar. Bei den am wenigsten resistenten Individuen ist auch eine Impfung von Spontantumoren erfolgreich. Die Entwioklung einer Impfgeschwulst wird verhindert nicht durch Antikörperbildung, sondern durch Atrepsie. Nur wenn die Avidität der Tumorzellen gestejgert oder bei den Körperzellen herabgesetzt ist, kommt es zur Geschwulstbildung. Und zwar kann es sich nicht bloss um die Aviditätssteigerung einzelner Zellen handeln, denn sonst müssten die Spontantumoren gleichfalls angehen. Die normale Immunität der Maus beruht auf atreptischer Basis und Borrel hat recht, wenn er sagt, ,die normale Avidität ist der Schlüssel des ganzen Krebsproblems". Die liünstliche Immunisierung gelingt nicht mit abgetötetem Geschwulstmaterial, dagegen durch Injektion ron avirulenten (hämorrhagischen) 'Tumoren. Derartig vorbehandelte Mäuse sind in $60-70$ pCt. inmun gegen virulente Tumoren. Hertwig und Poll erklären die Immonität durch die Auslese del normalen Giftfestigkeit der Mäuseindividuen, auch L. Michaolis leugnet die Immunität. Auch mit normalen Organen ist solche Immunität bis zu 80 pCt. zu erzielen. Doch sind, wie Borrel betonte, grosse Quantitäten von Impimaterial nötig, woraus sich die abweichenden Resultate von Hertwig und Poll (Stückchenimpfung) erklären. Bei diesen lünstlichen Immunisierungsversuchen ergab sich die auffällige und überraschende Tatsache der Erzeugung einer Immunität gegen alle Geschwälste, gleichviel welcher Art, sogar gegen das Chondrom, also eine Panimmunitat. Eine Immunität ist daher auch zu erreichen mit normalen Geweben (Blut, Embryonen, Milz, Brustdrüse). Ob dabei Antistoffe im Blate gebildet werden, ist zweifelhaft geworden, Versuche im Reagenzglas (Komplement) sind nicht befriedigend ausgefallen, E. will daher, da das Vorbandensein von Antikörpern überhaupt dubiös ist, auf diese Frage nicht weiter eingehen. Die Immunität durch Atrepsie erscheint besser fundiert, trotz der gegenteiligen Behauptungen von Hertwig und Poll sowie Gierke. Die abweichenden Resultate dieser Autoren erklären sich nach Ehrlich daraus, dass sie nicht mit maximal virulenten Tuworen gearbeitet. Der beste 'lumor von Hertwig und Poll ist. von keiner erheblichen Virulenz und Gierke hat mit den hämorrhagischen, also mit den notorisch schwächsten T'umoren gearbeitet. Für das Mass der Avidität ist nicht die Schnelligkeit des Wachstums, nicht das Gewicht des erzeugten'Tumors, sondern die Angangsziffer beweisend. Bei Verimpfung mit maximal virulenten, höchst aviden, exhaustiv and ereptiv wachsenden Tumoren erhält man bei Nachimpfung fast kein positives Resultat. Eine Hemmung wird auch durch schwächer virulente bewirkt und in diesem Sinne sind die Experimente von Hertwig und Poll nach Ehrlich geradezu eine Beweis für seine Anschauung. Die von Ebrlich gefundenen Tatsachen sind von Borrel bestätigt worden, Borrels Erklärung der Verhinderung des zweiten Tumors infolge der Bildung eines Antikörpers durch Resorption des zugrunde gegangenen Impfmaterials vermag Ehrlioh nicht gelten zu lassen. Zur Widerlegung dieser Anschauung hat Ehrlich verschiedene Versuche ausgeführt, einmal indem er Carcinom mit dem avideren Sarkom gemischt injizierte, dabei wurde ersteres von letzterem überwuchert, wolern nicht die Wachstumsenergie des Sarkoms durch verschieden langen Aufenthalt auf Eis abgeschwächt war, sodann indem el die Tumoren singulär nacheinander injizierte. Dabei wurde bei Vorimpfung mit Sarkon und Nachimpfung ron Carcinom das Carcinom 
verhindert. Bei Vorimpfung mit Carcinom und Nachimpfung von Sarkom wurde das Carcinom retardiert, während das nachgeimpfte Sarkom sehr starkes Wachstum zeigte. Durch letzteren Versuch hält Ehrlich die Atrepsio für unwiderleglich kewiesen. In praktischer Hinsicht ergibt sich daraus die Folgerung, auf eine Steigerung der natürlichen Resistenz nnd nicht auf die Erzielung von Antikörpern hinzuwirken.

Als dritter Referent bespricht hierauf Herr Lubarsch (Dïsseldorf) die Genese des Carcinoms beim Mensehen.

Für die erste Entstehung der Krebse des Menschen hat uns die experimentelle Krebsforschung keine Anbaltspunkte geliefert, auch dürfen ihre sonstigen Resultate nicht ohne weiteres auf die menschlichen Tumoren übertragen werden. Bei der Begriffsbestimmung des Carcinoms des Menschen als eines destraierend wachsenden Epithelioms können die Mäusekrebse überhaupt nur sehr bedingt zum Vergleiche herangezogen werden. Wir sind daber immer noch auf eine einzige Untersuchungsmethode - die histologische - angewiesen, die deswegen so erschwert ist, weil bei obiger Definition die ersten Anfänge der epithelialen Wucherung als carcinomatös mit Siclerheit nicht erkannt werden können. Die Verzweiflungstheorien von Adamkiewicz, Pfeiffer und Kelling, die die Entstehung des Carcinoms ron artfremdem Zellen ableiten wollen, sind ohne weiteres abzulehnen, die Stickersche Theorie, die die Krebszellen von artgleichen aber körperfremden Zellen ableiten will, steht auf der gleichen Höhe, wie die Wyville-Thom sonsche Theorie, die die Entstehung des Lebens auf der Erde durch auf Meteorsteinen zugeführte Keime erklären will, für die Erklärung der Entstehung des Lebens überhaupt. Eine ausführliche Besprechung widmet Lubarsch sodann der Theorie Ribberts mit dem Ergebnis, dass der mit soviel Arbeit und Scharfsinn durchgeführte Versuch Ribberts, das destruierende Wachstum ans der morphologischen Loslösung des Epithels durch entzündliche Veränderung des subepithelialen Bindegewebes zu erklären, als gescheitert zu betrachten ist. Der Beweis, dass es sich bei den als beginnende Carcinome gedeuteten Fällen wirklich um solche gehandelt hat, ist nicht erbracht worden, ebensowenig ist bewiesen, dass derartige entzündliche Veränderungen ausnahmslos bei beginnenden Carcinomen vorhanden sind. So hat z. B. Borrmann bei subepidermoidalen Tumoren, Lubarsch und Marckwald bei submukösen derartige Veränderungen vermisst. Die zellige Umwandlung des Bindegewebes ist nach Lubarsch auf Schädigungen von aussen zurückzuführen und kann daher keine Vorbedingung für die Genese des Carcinoms sein. Weiter entsteht die Frage, warum stellt sich der physiologische Zusammenhang zwischen Epitbel und Bindegewebe nicht wieder her? Warum sind ferner gerade die organoiden Tumoren die bösartigsten? Ueberhaupt erscheint die Anpassungshypothese Ribberts unverständlich für die Erliärung des destruierenden Wachstums. Aber selbst wenn die bisher besprochenen Annabmen Ribberts richtig wären, würde das Rätsel der nahezu unbegrenzten und schrankenlosen Wucherungsfähigkeit der Carcinomzellen nicht gelöst sein.

Das Carcinom entsteht also durch eine, nicht erst sekundär rom Bindegewebe aus erzengte primäre Veränderung des Epithels, die sich zunächst in einem Tiefenwachstum kund gibt. Bei Untersuchungen in dieser Hinsicht muss berüclssichtigt 
werden, dass kleine Carcinome nicht unter allen Umständen beginnende Carcinome sind. Die Frage: gibt es Veränderungen an den Zellen, die für Carcinom charakteristisch sind, muss vorlänfig dahin beantwortet werden, dass uns alle bisher beschriebenen Zell- und Kernveränderungen (asymmetrische Kernteilungsfiguren [v. Hansemann], Polymorphie der Zellen [Borst], heterotype Mitosen [Bashford und Murray]) im Stiche lassen, da sie sich nur bei vorgeschrittenen Tumoren finden. Auch alles, was unter dem Namen der Anaplasie (v. Hanse$\operatorname{man}$ n) zusammengefasst wird, ist noch nicht bei kleinen Carcinowien vorhanden. Infolge des Nachweises der multizentrischen Entstehung muss die Nachbarschaft der Carcinome mehr untersuebt werden, wobei zn unterscheiden ist zwischen Carcinomen, die von nicht verlagertem, und solchen, die von verlagertem Epithel ausgehen (subepitheliale und submuköse Carcinome). Die zellige Umwandlung des Bindegewebes muss dem Tiefenwachstum des Epithels nicht vorangehen, sondern kann vorhanden sein oder fehlen. Oft handelt es sich auch um kein kontinuierliches Tiefenwachstum, sondern um eine Art von Aufsplitterung der tieferen Epithelien, wodurch der Anschein eines altiven Loslösens und Eindringens des Epithels erweckt wird. Der häufigste Fall ist die Entstehung der Krebse von den Epithelien der Ausfübrungsgänge ans, der seltenste der von dem sezernierenden Epithel oder Parenchym der Organe. Letzteres ist überhaupt nur sicher bei der Leber und der Mamma nachgewiesen. Zuzugeben ist, dass die Bindegewebsreränderungen das Tiefenwachstum des Epithels erleichtern.

Obwohl gewichtige Gründe für die von Borst und $\mathrm{Sch}$ walbe angenommene angeborene Abnormität der Carcinomzellen sprechen (Vorkommen angeborener Blastome, T'eratoide mit destruierendem Wachstum, Naevi, Xeroderma, branchiogene Carcinome, Hypernephrome, subepidermoidale und submuköse Tumoren, heterologe Kankroide usw.), so ist es doch nicht wahrscheinlich, dass die biologischen Veränderungen des Krebsepithels stets auf primärer angeborener Ab. normität beruhen; auch geht es nicht an, die Carcinome als einseitig entwickelte Teratome zu betrachten. Doeh bestehen auch bei den Carcinomen Beziehungen zu Entwicklungsstörungen, die besonders in einigen Organen (Hoden, Ovarien, Nieren) deutlich sind. Aber sowohl die Beobachtungen am Menschen, wie die Ergebnisse der Krebsimplantationen bei Tieren sprechen dafür, dass die Eigenschaften des destruierenden Wachstums mit all ihren Folgeerscheinungen in höchster Ausbildung erst allmählich erworben (herangezüchtet) werden und mit abbängig sind von dem Verhalten der Umgebung und des Gesamtorganismus.

\section{Vorträge.}

1. Herr Karl Lewin (Berlin): Ueber Veränderungen eines Adenocarcinoms der Ratte bei dex Transplantation.

Ein primäres Adenocarcinom der Ratte gibt in der IIl. Ceneration zur Entstehung eines Kankroids, in der V. Generation zur Bildung von Sarkomen Anlass. Ein primärer Mischtumor ist ausznschliessen. Es kann zur Erklärung der nen entstandenen Geschwulstbildungen nur zwei Möglichkeiten geben: Entweder ist eine Metaplasie der primären Drüsenlrrebszellen zu Plattenepithel bzw. Bindegewebs- 
zellen zustande gekommen oder aber es haben die primären Drüsenkrebszellen bei weiterer Impfung die Epidermis bzw. die Bindegewebszellen der geimpften Tiere zu bösartigen Wucherungen angeregt. Vortr. neigt der letzteren Erklärung zu, für die er insbesondere in auch sonst bei seinen Tumoren beobachteten Epidermiswucherungen eine Stütze sieht. Die Sarkome leitet er wegen der Variabilität der Zellformen von den Endothelien her.

Selbstbericht.

2. Herr Thorel (Nürnberg): Kasuistiscbes zum Kapitel des sogenannten Mäusecarcinoms.

Vortragender bringt weitere Beobachtungen über die unter seinen Versuchsmäusen aufgetretene Krebsendemie. Während des Jahres 1907 konnte in jedem Monat, mit Ausnabme von 2 Monaten, das Auftreten von Spontantumoren beobachtet werden. Im ganzen fanden sich deren 14. Davon waren 12 bei sogenannten "Nullern" (Ehrlich) aufgetreten. Da die Impfung bei diesen an der Sehwanzwurzel vorgenommen war, die Tumoren aber an weit davon entfernter Stelle auftraten, so ist ein Zusammenhang mit der Jmpfung auszuschliessen. Ausserdem zeigten sich auch histologische Verschiedenheiten zwischen den zur Impfung verwandten und den aufgetretenen Spontantumoren. Für das endemische Auftreten vermag Th. keine Erklärung zu geben. Zusammenleben von gesunden mit Tumormäusen führte nicht zur Entstehung von Tumoren bei ersteren. Ob Wanzen oder andere Ektoparasiten event. eine Rolle dabei spielen könnten, liess sich nicht eruieren. Eine erhöhte Disposition durch Blutsverwandtschaft oder bei Geschwulstelternjungen war nicht konstatierbar. Das Auftreten der Spontantumoren hatte immer gewissermassen etwas Explosionsartiges an sich. Durch Kreuzungsversuche mit Mäusen, denen Tumoren exstirpiert waren, wurde eine Nachkommenschaft erzielt, die tumorfrei blieb.

Metastasenbildung fand Th. bei seinen Tumormäusen in den Lungen makroskopisch selten, mikroskopisch häufiger, doch hat man sich vor Verwechslungen mit adenopapillomatösen Spontantumoren, die bei den Mäusen ziemlich häufig in den Lungen vorhanden sind, zu hüten. Gelegentlich fand Th. Rückbildung der Geschwulstemboliepfröpfe in den Lungenarterien, ähnlich wie das M. B. Sch midt rom Menschen beschrieben. Zum Schluss mahnt Vortr. noch zur Vorsicht bei der Beurteilung der sog. Rückschläge und Umwandlungen (Adenom in Carcinom und umgekehrt), besonders in Hinsicht auf therapentische Effekte, da beides recht häufig schon in Spontantumoren nebeneinander vorhanden.

3. Herr Gierke (Berlin): Der Einfluss von Rasse und Herkunft der Mäuse a af die Uebertragbarkeit des Mäusekrebses.

Die von Ehrlich und Apolant, den englischen Autoren, Jensen, Michaelis u. a. konstatierten Verschiedenbeiten bei Transplantationen von Tumoren auf Mäuse anderer Länder, von zahmen auf wilde Mäuse und vice versa sind van 0 . Hertwig und $\mathrm{Poll}$ in Abrede gestellt worden. Demgegenüber konnte Gierke konstatieren, dass Mäuse fremder Länder weit weniger empfänglich sind als autochthone gegenüber ebensolchen Tumoren. Bei seinen Experimenten erzielte er 
mit Plattenepithelkrebs (englischer

Herkunft)

bei englischen Mänsen bei deutsch. Mäusen

nit hämorrhagischen Tumoren

mit Adenocarcinomen . . . .

in $57 \mathrm{pCt}$.
" 38 "
$n 75 "$

in 9 pCt. Impferfolg

$n 14$.

$, 21, "$,

Rassendifferenzen müssen hier entschieden obwalten. Auch Mäuse aus verschiedenen Zuchtanstalten verbielten sich verschieden. Nach 3 monatigem Aufenthalt in Kopenhagen waren deutsche Mäuse gegen die böchstvirulenten Ehrlichschen Tumoren ebenso unempfindlich geworden, wie dänische Mäuse das stets sind.

Jedenfalls ist bei Arbeiten mit ausländischen Mäusen Vorsicht am Platze und den Hertwig-Pollschen Ausführungen kein Vertrauen zu schenken.

4. Herr G. Herxhoimer (Wiesbaden): Ueber das Carcinoma sarcomatodes nud einen einschlägigen Fall des Oesophagus.

Es wird ein Carcinoma sarcomatodes im v. Hansemannschen Sinn (also Vermengung beider Geschwulstkomponenten, so dass das Sarkom das Stroma des Carcinoms darstellt) geschildert. Ist schon das Sarkom im Oesophagus sehr selten - 13 Fälle der Literatur - so ist ein Carcinoma sarcomatodes an diesem Fundort nur in einem Falle (v. Hansemann) bekannt, diese Tumorart überhaupt nur in etwa 20 Fällen. Die experimentelle Forschung kann uns gerade für das Carcinoma sarcomatodes mancherlei Schlüsse nahelegen. Nach diesen handelte es sich zameist wohl um eine sekundäre Entwicklung eines Sarkoms in einem Carcinom; in anderen Fällen mögen beide Geschwulsthomponenten von vornherein vorhanden sein. Diese beiden Formen hat Lippmann schon unterschieden. Vorliegender Fall wird in die erste einrangiert. Steht man auf dem Standpunkt, dass auch, im Falle das Sarkom später auftritt, dies schon entwicklungsgeschichtlich präformiert sein muss (im Experiment tritt die biologische Aenderung des Bindegewebes bei den zahlreichen Uebertragungen an die Stelle), so sind jene 2 Formen des Carcinoma sarcomatodes nahe verwandt. Hierauf weist auch die Aetiologie mit zwei getrennten Tumoren desselben Organs hin, welche zwar prinzipiell von dem Carcinoma sarcomatodes scharf geschieden werden müssen, aber praktisch oft nicht trennbar sind. Auch derartige multiple Tumoren werden ja, wie solche überhaupt, meist entwicklungsgeschichtlich erklärt. Lehren uns die Experimente gerade betreffs des Carcinoma sarcomatodes mancherlei, so ist vielleicht auch der Schluss berechtigt, diese Tumorart möchte auch beim Menschen häufiger sein, derartige Fälle aber nicht in diesem Stadium, sondern erst in dem des Reinsarkoms in die Erscheinung treten.

Selbstbericht.

5. Herr Schmorl (Dresden): Sarkomatöse Degeneration von Krebsmetastasen im Knochenmark.

Zur Erklärung der Häufigkeit der Knochenmetastasen bei primären Parenchymcarcinomen reicht nach Schm, die von v. Recklinghausen gegebene Erklärung nicht aus, vielmehr ist eine besondere Anziehung, ist das Vorhandensein von "Wuchsstoffen" im Knochenmark anzunehmen. Schm. schliesst das aus Fällen, wo Organmetastasen fehlten, wo aber überall, wo Knochen vorhanden; z. B. im 
Kehlkopf und sogar in beterotopen Knochenbildungen (Knochenplatten in der Aortenwand), Metastasen vorbanden waren. In einem solchen Falle fand Schm. in allen Knochen Carcinom kombiniert mit Osteochondrosarkom. Es handelte sich um einen 61jährigen Mann, der ein Jahr vor seinem Tode an neuralgischen Schmerzen erkrankte, dann eine Spontanfraktur erlitt. Bei der Section fand sich ein osteoplastisches Prostatacarcinom mit Auftreibung sämtlicher Knochen. Die Lungen zeigten linochenharte Metastasen, die teils carcinomatös, teils osteochondrosarkomatös, teils gemischter Natur waren. In sämtlichen Knochen fand sich kombiniert Carcinom mit Osteochondrosarkom und zwar in einer Anordnung, dass das erstere von letzterem erdräckt zu werden schien. Da eine diffuse Osteochondrosarkomatose sämtlicher Knochen bisher noch nicht beobachtet, so ist nach Schm. die Annahme von zwei Primärtumoren abzulehnen und das Osteochondrosarkom als unter dem Einfluss der Carcinommetastasen des Prostatacarcinoms entstanden anzusehen. In zwei anderen Fällen fand Schm. ähnliche Bilder.

6. Herr A. Albrecht (Wien): Uebor Chorionepitheliom and verwandte Geschwülste.

Der Vortragende bespricht histologische Befunde in gewissen Hoden- und Schilddrüsentumoren, welche im Sinne der Sarkocarcinome zu deuten sind. Es bandelt sich hier in erster Linie um chorionepitheliomartige Hodengeschwälste, bei denen man nachweisen kann, dass die Langhans-zellähnlichen Formationen epithelialer Herkunft sind und der carcinomatösen Komponente entsprechen, während sich die Syncytien von der Bindesubstanz ableiten und als sarkomatöse Komponente aufzufassen sind. Bei sehr vielen malignen Hodentumoren, insbesondere aus der Gruppe der grosszelligen Carcinome, sieht man insofern ein eigentümliches Verhalten des Stromas, als von den gröberen alveolären Bindegewebssepten aus sich ein feineres Netzwerk in die Epithelzellkomplexe eindrängt. Dieses Netzwerk besteht entweder aus kleinen Bindegewebszellen oder grösseren Spindelzellen oder auch, manchmal reichlicher, manchmal spärlicher, aus Syncytien. Es ist hier somit die histogenetische Erklärung der chorionepitheliomartigen Hodentumoren (und wahrscheinlich aller in anderen Organen [Magen, Leber, Zirbeldrüse usw.]) bcobachteten Chorionepitheliome angegeben.

Daran reiben sich die Befunde von 6 Fällen von Sarkocarcinomen der Schilddrüse, welche ebenfalls zeigen, dass das eigentliche Agens der Geschwulstbildung die epitheliale Wucherung ist, welche auf dic Bindesubstanz (einschliesslich der Gefässelemente) produktiv einwirkt, so dass mitunter histologisch ganz sarkomatöse Formen entstehen. In manchen Tumoren kann eine derartige Mischung der carcinomatösen und sarkomatösen Komponente eintreten, dass man die grösste Mühe bat, die einzelnen Formen auseinander zu halten.

Die Art der Metastasenbildung in dem einen Falle macht es wahrscheinlich, dass auch Anteile der sarkomatösen Komponente ebenso wie die der carcinomatösen verschleppt werden können.

Die histologischen Bilder dieser Tumoren sind vielfach mit denen der Sarkocarcinome der Mäuse und Ratten vollkommen identisch. Auch in der normalen Histologie findet sioh in einigen Organen (Speicheldrüse, Rete des Hodens) vergleichbares Verhalten zwischen Epithel und Bindegewebe. 
504 Koch, Aus den Verbandlungen der Deutschen Pathologischen Gesellsehaft.

Die Untersuchungen an einer grossen Reibe von Chorionepitheliomen haben den Vortragenden zu der Meinung geführt, dass es sich auch bei diesen Goschwülsten um ähnliche Histogenese handelt. Bei ihnen ist das biologische Agens die destruierend wuchernde Langhans-Zelle. Die sarkomatösen Komponente sind durch Syncytien vorgestellt. Dieselben stammen teils vom mütterlichen Gewebe, wo der Tumor infiltrierend wächst, teils von der Deckschicht der Zellen, welche gleichzeitig mit der Langhans-Zellschicht proliferiert. Da junge menschljehe Eistadien (Peters Ei and ein etwa 2 Wochen altes menschliches Ei in situ) keine der Annahme der Entstehung der Deckschicht aus Bindesubstanz widersprechenden Befunde ergeben, im Gegenteil dieselbe unbedingt stützen, ist auch diese Prolifcration im Sinne der sarkomatösen Komponente aufzufassen. Dass sich aber trotzdem an gewissen Stellen und in gewissen Stadien echte epitheliale Syncytien aus Langhans-Zellen bilden, soll nicht geleugnet werden. Auch bei Chorionepitheliomen, namentlich bei der atypischen Form, findet sich oft ein unentwirmbares Gemenge beider Zellarten. Aehnliches findet auch in den Zellsäulen junger Eistadien statt, wie besonders Mallorg-und Bielschofsky-Präparate beweisen.

Selbstbericht.

7. Herr Versé (Leipzig): Ueber die Histogenese der Schleimhatcarcinome.

Auf Grund ausgedehnter Untersuchungen an sehr zahlreichen gutartigen und bösartigen Tumoren des Magen-Darmkanals kommt der Vortr. zu dem Resultat, dass die primäre Veränderung der Epithelzelle das ausschlaggebende Moment für die Entstehung des Carcinoms sei. Die Umwandlung der Epithelzelle in eine Krebszelle erfolgt allmählich, stufenweise; im Magen-Darmkanal knüpft die Krebsbildung gern an bereits bestehende adenomatöse oder polypöse Wucherungen an.

Das Wachstum des ausgebildeten Carcinoms erfolgt meist allein durch die Ausbreitung der Krebszellen selbst; nur sehr selten gelingt es, mit Sicherheit in der Nachbarschaft grösserer Carcinome das Auftreten primärer kleiner Krebsherdchen nachzuweisen. Dass in der Tat isolierte Epithelumwandlungen in Carcinomzellen am Rande älterer Krebse rorkommen, ist nicht zu bezweifeln und wird u. a. durch eine Beobachtung des Vortragenden selbst wieder bewiesen, welcher inmitten einer langen Schnittserie eine zirkumskripte Umwandlung des Epithelbelags in der anstossenden Schleimhaut eines carcinomatösen Ulkus des Rekturn demonstrieren konnte.

Selbstbericht.

8. Herr Sticker (Berlin): Sarkom und infektiöses Granulom bei Hunden.

St. demonstriert zahlreiche Präparate seines experimentell übertragbaren Rundzellensarkoms vom Hund. Von Bashford sind diese Stickerschen Hundegeschwülste als infektiöse Granulome angesprochen worden, nicht als echte Tumoren und zwar, weil sie erstens durch den Koitus übertragbar sind und zweitens histologisch sich wie solche Granulome verhielten. Diese Einwände sind nach St. zurückzuweisen. Bei dem langdauernden Koitus der Hunde werden Verletzungen gesetzt, die Transplantationen ermöglichen. Histologisch feblen in den Tumoren die für die Lymphozyten des Hundes charakteristischen fuchsinophilen Granula 
(Schridde). Ferner spricht gegen diese Ansicht das Freibleiben der Lymphdrüsen und die 3 Monate dauernde Inkubationszeit. In den Lymphdrüsen finden sich nur Metastasen, wenn ulzeriertes Material zur Impfung verwandtwurde, wobei gleichzeitig infektiöses Material eingebracht wurde.

\section{Diskussion zu den Referaten und Vorträgen $1-8$.}

Herr Sticker findet den Versuch der Diskreditierung der Ehrlichschen Versuche merkwürdig, die abweichenden Resultate von Hertwig and Poll erklären sich daraus, dass diese Experimentatoren in die "Tumorzone" geimpft haben, statt in die Immunzone zu impfen. Betreffs der Antikörper teilt er die Ehrlichschen Auffassungen.

Herr Gierke betont Ehrlich gegenüber, dass er bei seinen Versuchen wohl von hämorrhagischen Mäusetumoren ausgegangen, dass er aber auch mit anderen Tumoren experimentiert hat. Maximal virulente Tumoren bieten nach G. nicht immer die Erscheinungen atreptischer Immunität, ohne dass er damit die Ehrlich schen Resultate anfechten möchte. $\mathrm{Zu}$ unterscheiden ist zwischen maximal virulenten Tumoren, die eine Nachimpfung ausschliessen, und solchen, die diese begünstigen. Der Grund für die verschiedenen Resultate der englischen Krebsforscher und Ehrlichs ist nach G. möglicherweise im Impfmodus zu suchen. Ehrlich verwendet relativ grosse Mengen Impfmaterial, wodurch die Grundlagen für die Immunität überimpft werden. Für sehr wichtig hält Gierke den Austausch von Tumormaterial zwischen London und Franlifurt a. M.

Herr Apolant gibt einen gewissen durch den Impfmodus bedingten Unterschied zu. Bei der Beurteilung der Resultate ist eine Wägung der erzielten Tumoren nötig. A. weiss nicht, woran es liegt, aber im Ehrlichschen Institut wird jedesmal atreptische Immunität erzielt.

Herr Sternberg: Trotz der wohl nun anbrechenden "Aera der Sarkocarcinome" hält St. inbezug auf die von ihm beschriebenen Hodentumoren an der Auffassung dieser Geschwülste als peritheliale Sarkome fest.

Herr Lewin hat bei seinen Rattenversuchen bei Nachimpfungen vielfach Tumoren erzielt, gewogen hat er sie allerdings nicht.

Herr Schmorl führt zwei Fälle aus der menschlichen Pathologie an, wo bei nacheinander bei demselben Individuum entstandenen Tumoren keine Beinflussung aufeinander zu konstatieren war. In dem ersten Falle handelte es sich um ein Individuum, bei dem eine melanotische Geschwulst des Gesichts durch Röntgenbestrablung beseitigt war und das später an Magenkrebs erkrankte, bei dem zweiten um Kombination von Lymphosarkom mit einem, wie durch die klinische Beobachtung nachgewiesen, später entstandenen Uteruscarcinom. In beiden Fällen waren bei der Sektion gleichgrosse Metastasen von beiden Tumoren vorhanden. Ferner teilt Schmorl - wegen der Seltenheit derartiger Befunde - noch mit, dass er neuerdings ein Magencarcinom beim Kaninchen und einen doppelseitigen sarkomatösen Lungentumor bei einem 17 Jahre alten Kanarienvogel gefunden habe. Erage.

Herr Thorel stellt eine einen Doppeltumor bei seinen Mäusen betreffende 
Herr Marchand nimmt in einem Falle von Nebennierentumor, wo er Metastasen ron sarkomatösem Charakter beobachtete, an, dass die Spindelzellen aus epithelialen Zellen hervorgegangen seien. Ohne verallgemeinern zu wollen, möchte er doch auf diese Möglichkeit der Entstehung der bereits besprochenen 'Tumoren hinweisen.

Herr R. Meyer spricht die Befürchtung aus, dass man nun mehr denn je dem "Phantom des beginnenden Carcinoms" nachjagen werde. Auch ohne dass destruierendes Wachstum vorliegt, kann man die praktische Diagnose Carcinom stellen, z. B. bei beginnenden Carcinomen des Ovariums. Bei Tumoren des weiblichen Genitaltraktus sah M. häufig Umwandlungen von epithelialen Elementen in solche ron Spindelform, doch ist die letztere nicht für Bindegewebe beweisend.

Herr Löwenstein beobachtete bei Scharlachölinjelitionen in die Parotis des Kaninchens, dass epitheliale Elemente in Spindelzellen übergingen. Mit der Färbung nach Mallory liess sich eine gewisse Differenzierung bewirken.

Herr Saltykow berichtet über ein malignes Myom mit epithelialen Einschlüssen und führt verschiedene Tumoren auf, bei denen er Umwandlung von epithelialen Elementen in Spindelzellen beobachten konnte.

Herr Lubarsch bezweifelt, dass es sich bei den Präparaten von Herrn Lew in um eine Umwandlung in Sarkom handelt und gibt das Sarkom höchstens für das letzte der aufgestellten Präparate zu. Auch bei den Präparaten Apolants erliennt er noch Stroma zwischen den spindeligen Zellon, die er noch für epitheliale hält. Aehnliche Zweifel äussert er bezüglich der Ehrlichschen Präparate. Ehrlichs Standpunkt zur Atrepsie habe sich etwas geändert, man liönne jetzt nur noch von relativer Atrepsie reden. Herr L. warnt vor einer Verallgemeinerung der Atrepsielehre.

Herr $\Lambda$ lbrecht bleibt gegenüber Sternberg auf dem Standpunkt stehen, dass die fraglichen Hodentumoren epithelialer Natur sind und will sie nicht als peritheliale Sarkome bezeichnet wissen. Er beruft sich auf ähnliche Bilder bei der Einbettung junger menschlicher Eier.

Herr Henke teilt einige Beabachtungen beim Mäusekrebs mit, auf dessen Bedeutung für die Krebsforschung er erneut hinweist. Drüsenmetastasen hat er beim Mäusekrebs nicht gesehen. Das Adenom der Mäuse sei nicht rom Krebs zu trennen.

Herr Marchand spricht sich für die epitheliale Natur der Hodentumorzellen aus, betont aber die grosse Schwierigkeit, die Natur der Zellen an manchen Orten zu erkennon.

Herr Lewin bält an der Sarkomnatur seiner Rattentumoren fest.

Herr Beneke berichtet über einen Fall von Carcinoma sarcomatodes, bei dem später der sarkomatöse Charakter des Stromas verloren ging. Bezüglich der Carcinomgenese glaubt Herr B., dass der Krebs nicht nur aus sich heraus, sondern auch durch carcinomatöse Umwandlung der Nachbarschaft wächst.

Herr Gierke betont Herrn Henlie gegenüber, dass die Mäusckrebse auch Drüsenmetastasen machen können. 
Herr Orth: Es seien zwei verschiedene Dinge hier besprochen worden, der Mäusekrebs und der Menschenkrebs. Beim Mäusekrebs sei man aber ganz auf die Genese des Sarkoms und der Metastasen abgekommen, die Genese des Krebses selber sei gar nicht erörtert. Wie Herr Henke, erkIärt auch er die Trennung des Adenoms vom Krebs der Mäuse nicht für gerechtfertigt. Auf die Diskussion über den Menschenkrebs könne man das Reutersche Wort anwenden: "Die grosse Armut in der Stadt kommt yon der grossen Powerteh her"; es sei wohl die formale Genese besprochen, die kausale aber nicht gefordert. Der Befund von Glykogen in den Epidermiszellen, da, wo der Krebs gegen die Haut wächst, deute lediglich auf eine Vermehrung der Epithelzellen; man dürfe den Vorgang als hyperplastisch bezeichnen, aber nicht ohne weiteres als lrebsig.

Herr Sticker ist misscerstanden worden. Er stimmt duichaus der zweiten These von Herrn Lubarsch zu, nur hat er als eine der Ursachen des Carcinoms die Implantation artgleicher aber körperfremder Zellen bezeichnet.

Herr A polant (Schlosswort): Die Ehrlichsche Schule mache keinen prinzipiellen Unterschied zwischen Adenom und Carcinom, doch sei hervorzuheben, dass die Ausgangstumoren stets adenomatöse Struktur haben. Bezüglich der Immunisierungsresultate sei za bemerken, dass sich bei den vorbehandelten Tieren nicht nur quantitative, sondern auch qualitative (histologische) Unterschiede der etwa noch auftretenden Geschwulstknötchen gegenüber nicht vorbehandelten Tieren fänden. Herr Lubarsch habe ihn missverstanden; er habe zwei ganz verschiedene Dinge demonstriert, 1. die Umwandlung des Stromas in Sarkom, ohne die geringste Beteiligung der Krebsnester, 2. die Umwandlung der Epithelzellen zu spindeligen Gebilden. Gegenüber Herrn Gierke stellt er fest, dass Rassenunterschiede der Mäuse wohl eine Rolle spielen können, aber nicht müssen. Endlich verteidigt A. nochmals ausführlich die Atrepsie. Eine absolute atreptische Immunität sei ron Ehrlich nie behauptet worden. Auf den Menschen dürfe die Theorie selbstverständlich nicht ohne weiteres angewandt werden.

Herr Lubarsch (Schlusswort): Er habe bei den Ursachen des Carcinoms unterschieden 1. die biologischen Veränderungen des Epithels, 2. die allgemeinen und lokalen Widerstände. Untersuchungen über den allerersten Beginn des Carcinoms und damit über ihre Genese scheitern an der grossen Schwierigkeit, was man bereits als Carcinom ansehen soll. Die Forschungen über den Mäusekrebs hält L. doch für sehr bedeutungsvoll für den Krebs des Menschen. Zur Erklärung der Genese gewisser Krebse, besonders der Mamma, glaubt L. annehmen zu sollen, dass aus ein und derselben Zelle Zylinderzellen, Epidermiszellen und Bindegewebe hervorgehen können. Prinzipiell möchte L, auch ein infelitiöses Agens für die Carcinomgenese nicht ablehnen.

\section{Herr Löhlein (Leipzig): Cystisch-papillärer Lungentumor.}

Ein im rechten Unterlappen einer 69 jährigen Fran gefundener cystisch-papillärer Tumor mit praller Schleimfüllung der Alveolen und Bronchiolen des veränderten, übrigens vollkommen entwickelten Bezirks der Lunge, wird von L. mit multiplen Tumoren von sehr ähnlichem Bau, die Helly beschrieben hat, für wesensgleich angesehen. L. führt die pathologische Bildung mit Wahrscheinlich- 
keit auf Ausbleiben der Entfaltung des betr. Lungenabschnittes (fötale Atelektase) im Sinne Hellers zurück.

Selbstbericht.

10. Herr Simmonds (Hamburg): Ueber das Vorkommen ron Spirochaten in zerfallenen Carcinomen.

Vortr. fand Spirochaeten niemals in geschlossenen, selten in einfach ulzerierten, häufig in gangräneszierenden Krebsen. Angetroffen wurden sie nur dort, wo eine Infektion mit Mundbakterien möglich war und da die Spirochaeten der Carcinome auch in Form und Grösse mit denen der Mundhöhle übereinstimmen, hält er sie für Abkömmlinge der Mundflora. Sie sind in den oberflächlichen Krebsschichten mit anderen Bakterien reichlich vermischt, wuchern in der etwas tieferen Schicht reichlicher und finden sich hier oft in Reinliulturen in dichten Geflechten, wahrscheinlich, weil sie anaerob besser gedeihen. Sie sind zum Teil Schuld an dem Fötor der Tumoren, können in Gefässe eindringen und so in andere Organe verschleppt werden, sind aber sonst harmlose Schmarotzer. Ihre Bedeutung liegt in der Schwierigkeit, sie von der Spiroohaete pallida zu unterscheiden. Es ist daher grosse Vorsicht geboten in der Deutung des Spirochaetennachweises in zerfallenen, der Syphilis verdächtigen Geschwülsten.

Selbstbericht.

\section{Diskussion:}

Herr Schmorl fieut sich, dass Herr Simmonds seine Untersuchungen bestätigt. Er selbst hat Spirochaeten auch mehrfach in metastatischen Abszessen gefunden. Die Spirochaeten winden sich gern durch die Zylinderepithelien der Bronchien hindurch, ähnlich wie Spirochaete pallida bei angeborener Syphilis.

Herr Fraenkel berichtet über ähnliche Befunde. Er kann die Spirochaeten beim Krebs und bei Syphilis nicht sicher unterscheiden.

Herr Sternberg hat einen analogen Fall wie Herr Löhlein beobachtet, mit Metastasen an den basalen Hirnnerven.

Herr Deetjen hat bei allen Mäusetumoren, auch bei geschlossenen, Spirochaeten gefunden, und zwar nicht im Tumor selbst, sondern im umliegenden Bindegewebe und nur im frischen Präparat; sie waren etwa eine halbe Stunde lang beweglich. Bei Einwirkung von Aelherdämpfen rollen sie sich zu kleinen Kugeln (Ruheformen) ein. Die Spirochaeten fanden sich auch im Blut bei Krebsmäusen, wie bei gesunden Mäusen, und liessen sich auf andere Mäuse übertragen. Wahrscheinlich werden die Spirochaeten mit den Tumoren zugleich überimpft; ob sie etwas mit dem Tumor zu tun haben, ist fraglich.

Herr Saltykow berichtet über einen dem Löhlein schen äusserst ähnlichen Fall; hier fanden sich auch krebsige Stellen.

Herr Zieler: Die Syphilisspirochaeten haben zugespitzte Enden, die Carcinomspirochaeten nicht.

Herr Schmorl: Die Unterscheidung zwischen Spirochaeten mit spitzen und mit stumpfen Enden sei praktisch sehr schwer, namentlich in Schnittpräparaten. Er fragt Herrn Deetjen, ob er Spirochaeten beim Mäusekrebs in Silberpräparaten gefunden hat? 

funden.

Herr Thorel hat nie Spirochaeten in Silberpräparaten vom Mäusekrebs ge-

Herr Henke ebensowenig.

Herr Aschoff berichtet über Präparate von Gaylord, der ebenso wie Deetjen Spirochaeten in der Umgebung der Mäusekrebse gefunden hat, auch Phagozytose der Spirochaeten seitens der Krebszellen. haben.

Auch Gaylord glaubt, dass die Spirochaeten mit dem Krebs nichts zu tun

Herr Simmonds hat Spirochaeten nur zwischen den Epithelien gesehen, nicht in ihnen.

11. Herr Borrmann (Braunschweig): Zwei polypöse Oesophagussarkome bei einem Individuum.

Bei einem 50 jährigen Mann, der fast nichts mehr schlucken konnte und an Inanition gestorben war, fand sich $12 \mathrm{~cm}$ oberhalb der Kardia ein polypöser, wurstförmig sich im Lumen nach abwärts erstreckender Tumor, daneben ein kleinerer ähnlicher (Demonstration). Die histologische Untersuchung stellte nicht ganz klar, ob es sich um ein einfaches Sarkom oder ein Myosarkom handle.

\section{Diskussion:}

Herr Lubarsch: Bei Pferden und Rindern sind solche Tumoren häufiger, nur ist der Sitz höher.

Herr Orth fragt nach dem Verbalten der Schleimhaut an den T'umoren.

Herr Borrmann hat sie noch nicht untersucht.

Herr M. B. Schmidt hat einen ähnlichen Fall beobachtet, der wahrscbeinlich als Rhabdomyosarkom anzusprechen war. An der Basis einer der Geschwülste hatte sich ein Carcinom entwickelt.

12. Herr Stoerck (Wien): Zur Histogenese der Grawitzschen Geschw ülste.

S. bringt Argumente für die nephrogene Herkunft und solche gegen dic ursprüngliche von Grawitz angegebene hypernephrogene Deutung dieser Geschwülste. Man habe die Tatsache, dass solche Geschwülste immer wieder von der Niere und nicht von der Nebenniere ihren Ausgang nehmen, durch die Beziehung zwischen Heterotopie und Geschwulstwachstum zu erklären versucht. Dem sei entgegenzuhalten, dass ja auch Nebennierenversprengungen in andere Parenchyme (Leber, Geschlechtsdrüsen) recht häufig vorkommen, obne in entsprechender Häufigkeit zum Ausgang Grawitzscher Tumoren zu werden. Zur Erklärung des differenten Aussehens zwischen primären Nebennierengeschwülsten und den angeblich hypernephrogenen der Niere, wurde auf geänderte Ernährungsverhältnisse in der Niere und Aehnliches hingewiesen. S. konnte tierexperimentell zeigen, dass in die Niere verlagertes und dort wucherndes Nebennierenrindengewebe daselbst immer nur Bildungen nach Art der typischen Adenome der Nebennierenrinde formiere. Auf die Grawitzsche Argumentation eingehend, zeigt S. an der Hand eines grossen Materials, dass sich die Lokalisation einerseits der Tumoren, andererseits der Versprengungen nicht decke, indem letztere am häufigsten 
am oberen Nierenpol, erstere am häufigsten in der Nierenmitte, in zweiter Linie am unteren $\mathrm{Pol}$ und erst in dritter am oberen $\mathrm{Pol} z u$ finden seien.

Die Aehnlichkeit von Grawitztumorzellen mit den Zellen der Nebennierenrinde bestreitet $S$., insbesondere die Gemeinsamkeit der sogenannten Fettinfiltration. Die grossen Tropfen der Grawitzzellen seien nicht Fett, sondern das vakuolenartige Produkt einer hypertrophischen Degeneration. Die Fettröpfchen können den Tumorzellen völlig fehlen, niemals erfüllen sie, wenn äberhaupt vorhanden, in der für die Nebenniere so charakteristischen Weise gleichmässig das Protoplasma.

Eine Uebereinstimmung könne nur bezüglich der Form der Zellverbände im Sinne der gemeinsamen alveolären Zellgruppierung gelten. Aber gerade für die Form der Zellverbände ergäbe sich ein prinzipieller Gegensatz in der Lumenand Zottenbildung bei den Tumoren, für welche jegliches Analogon in der Nebenniere fehle.

Die von Grawitz vertretene Gegensätzlichlieit zwischen Tumor- und Nierenepithelform habe nur Geltung für den Vergleich mit physiologischem Nierenepithel; es lassen sich aber vollständige Uebereinstimmungen mit pathologischen Nierenepithelbildern konstatieren. Solche Epithelformen zeigen auch die Glieder der Reibe: cystisches NierenkanäLchen, papilläres Kystom, Zottengeschwulst und Grawitztumor der Niere. Diese Glieder kommen mehrfach vollzählig, oft auch im innigsten Nebeneinander, in ein und derselben Niere oder auch in den beiden Nieren desselben Individuums zur Ansicht.

Besonders zu bemerken wäre die Tatsache, dass nicht nur in Grawitztumoren häufig typische Zottenbildung vorkommt, sondern auch in typischen Zottengeschwülsten der Niere in umschriebener, mehr oder minder ausgedchnter Erstreckung Partien vom ausgeprägten Typus Grawitzscher Geschwülste.

13. Herr Marchand (Leipzig): Hyperplastische akzessorische Nebennieren im Pankreas und in der Niere, Demonstration.

Grawitzscher Tumor in Pankreas und in der Niere bei demselben Individuum; Metastasenbilaung ist hier nicht anzunehmen. M. ist von den Anschauungen Stoercks überrascht. Zwar gibt es Tumoren renalen Ursprungs, deren Zellen mit Nebennierenzellen Aehnlichkeit haben; indes muss für die echten Grawitzschen Tumoren an dem suprarenalen Ursprung festgehalten werden.

14. Herr Aschoff (Freiburg i. Br.): Untersuchungen des Herrn Dr. Cohn über Nebennieren und Herzhypertrophie.

Untersuchung von 98 Fällen. Nur etwa 30 pCt. der Fälle, in denen Herzhypertrophie vorhanden war, zeigten eine Hypertrophie des Nebennierenmarkes. 18 mal fanden sich in der Nebenniere Rundzellenanhäufungen, und zwar erwiesen sich diese Rundzellen als Lymphoblasten und Plasmazellen, nicht als Sympathikusbildungszellen. Versuche, durch Adrenalininjelitionen Herzbypertrophie hervorzurufen, hatten nur bei wiederbolten kleinen Injektionen einigen Erfolg, nicht bei einer einzelnen grossen Injektion. Untersuchung der Nebennieren bei so behandelten Tieren ergab nur unwesentliche Veränderungen.

15. Herr Robert Meyer (Berlin): Zur normalen und pathologischen Anatomie der akzessorischen Nebennierenrinde des Genitalgebietes.

M. fand die Knötchen meist in der Nähe der Keimdrüsen, nur einmal bei 
mangelndem Deszensus im Hoden selbst; sie werden beim Deszensus aus der Umgebung der Nebennieren oder von dieser selbst fortgeschleppt. Autochthone Nebennierenknötchen sind am Genitale nicbt bekannt: Picks Befunde sind Wucherungen des Serosaepithels, deren Entstehung unter entzündlichen Veränderungen M. in allen Stadien nachweisen konnte; sie enthalten weder Lipoide, noch nehmen sie jemals die Struktur der Zona fasciculata an.

Während die akzessorischen Knötchen der Nebennierenrinde bei Föten und Kindern nicht selten die Schichtung in zwei oder drei Zonen erkennen lassen, fehlt in vier Fällen bei Erwachsenen die Zona glomerulosa und reticularis. Diese wenig entwickelten Knötchen sind jedoch proliferationsfähig und können auch, wie in einem Falle bei Tubargravidität, hyperplasieren und zwar an der Peripherie, trotzdem die Glomerulosa (Polls Keimschicht) fehlt.

Ein Fall von "Ovarialtumor" gleicht völlig den Grawitzschen Tumoren und wird von M. als maligner Tumor aus akzessorischer Nebennierenrinde aufgefasst.

Selbstbericht.

\section{Diskussion:}

Herr Lubarsch bestreitet prinzipielle Unterschiede zwischen den Grawitzschen Nierentumoren und Nobennierengeschwülsten; auch in letzteren können Hohlräume beobachtet werden. Erfahrungsgemäss seien versprengte Nebennierenkeime und kleine Nierencysten häufig kombiniert, sodass auch diese Nierenepithelien durch wachsende Grawitzsche Tumoren zur Wucherung angeregt werden könnten, wodurch eine Art Mischtumor entstehe.

Herr Beneke macht darauf aufmerksam, dass in Grawitzsehen Tumoren gelegentlich deutliche Reste von Nebennierengewebe vorhanden sind, die allmählich in Geschwulstgewebe übergehen.

Herr Stoerck glaubt, dass letztere Bilder durch Einwachsen des Nierentumors in die versprengte Nebenniere zustande kommen. Den Marchandschen Fall hält or nicht für beweisend, da der Pankreasknoten als Metastase des Nierentumors aufzufassen ist. Der Glykogengehalt spricht sogar gegen die Nebennierennatur, da Rindenadenome der Nebenniere glyliogenfrei sind.

16. Herr Henke (Königsberg): Uterusmyom mit schleimhäutiger Innenfläche.

Demonstration eines polypösen Uterustumors, der aus einer Muskclwand und einer schleimhäutigen Innenfläche besteht; H. ist der Ansicht, dass es sich nicht um eine Missbildung, sonderm um eine Geschwulst handelt.

\section{Diskussion:}

Die Herren Schmorl, Gierle und Robert Meyer borichten über ähnliche Fälle.

17. Herr Zieler (Breslau): Ueber ein malignes Myom der Haut.

Demonstration von Präparaten eines Myoms von der Wange einer 67 jährigen Frau, das angeblich aus einer Warze entstanden war. Die Malignität dokumentierte sich durch Einbruch der Geschwulstzellen in Gefässe. 
512 Koch, Aus den Verhandlungen der Deutschen Pathologischen Gesellschaft.

\section{Diskussion:}

Herr Beneke beobachtete ein erbsengrosses (schmerzloses) Myom am Bein, das vielleicht als Myom der Arterienwand anzusprechen war.

Herr Lubarsch: Herr Zieler habe die Ausdrücke "malignes Myom" und "Myosarkom" promiscue gebraucht. Es sei aber scharf zu unterscheiden zwischen dem nalignen Myom mit Erhaltung der Myomzellenstruktur und dem Myosarkom mit Zellpolymurphie.

Herr Robert Meyer: Ganz reine maligne Leiomyome gibt es nicht, sie enthalten alle mehr oder weniger Sarkomgewebe.

Die Herren Orth, Dürck und Fraenkel berichten über maligne Myome, die nur aus glatter Muskulatur bestanden.

18. Herr Maresch (Wien) demonstriert einen cystischen Tumor des kleinen Beckens mit grossen papillären Wucherungen.

19. Herr Robert Meyer (Berlin): Demonstration einer bis in die Warzel des Mesokolon ausgedehnten heterotopen Epithelwucherung des Darmepithels.

In einem Falle von ulzeröser Striktur am Dickdarm mit Verdickung und Verbärtung des Mesoliolon dringt Oberflächenepithel, welches ulzerierte Stellen überhäutet, in Gestalt von Schläuchen in die Tiefe (Granulationsgewebe) und durch Submukosa unter grosser Ausbreitung daselbst in die Muskularis und ron dort in das Bindegewebe und die Lymphknoten des Mesokolon und zwar in zerstreuten Zügen längs der Lymph- und Blutgefässe, mit einschichtigem Epithel und einem breiten Mantel zellreichen Bindegewebes. Besondere Strukturabweichungen des Epithels fehlen. Die Aehnlichkeit mit den heterotopen Epithelwucherungen am Uterus und besonders an den Tuben wird hervorgehoben, welche auch zuweilen bis in das Lig. latum vordringen. - Zur Annahme einer malignen Neubildung liegt morphologisch und nach dem klinischen Verlauf kein Anlass vor.

Selbstbericht.

\section{Diskussion:}

Herr Orth fragt, ob Schleim in diesen Drüsen enthalten gewesen sej, was Herr Meyer verneint. 